the mixture rose from $61^{\circ}$ to $67^{\circ}$. The resulting mass was washed repeatedly with alcohol without becoming white, but it crystallized from boiling ligroine in minute white needles, turning brown in contact with the air.

\title{
Note on Thiocarbanilide.
}

It was found that the compound $2 \mathrm{C}_{8} \mathrm{H}_{7} \mathrm{~N}$. $\mathrm{HgCl}_{8}$ underwent rapid decomposition when exposed in contact with carbon disulphide to the action of sunlight. Subsequently, that this decomposition took place merely by heating, and could be best effected in the following manner :

The purified chloride of diphenylnercurammoninm was dissolved in alcohol, and after being mixed with carbon disulphide, was boiled for several days under a return cooler. When the reaction was finished, which was made evident by the clearing of the liquid, the precipitated $\mathrm{HgS}$ was filtered off, and the filtrate set aside to crystallize.

After a second crystallization, a pure product was obtained with a melting point of $144^{\circ}$, and which proved on analysis to be thiocarbanilide, $\mathrm{C}_{13} \mathrm{H}_{12} \mathrm{~N}_{2} \mathrm{~S}$.

\begin{tabular}{|c|c|c|}
\hline & Found. & Theory. \\
\hline Carbon... & 68.31 & 68.42 \\
\hline Hydrogen & 5.41 & 5.26 \\
\hline Sulphur.. & 14.00 & 14.04 \\
\hline
\end{tabular}

Thiocarbotoluidide and thiocarboxylidide were formed in the same manner from $2 \mathrm{C}_{7} \mathrm{H}_{9} \mathrm{~N} . \mathrm{HgCl}_{2}$ and $2 \mathrm{C}_{8} \mathrm{H}_{11} \mathrm{~N} . \mathrm{HgCl}_{2}$.

The cyanide of diphenylmercurammonium also yields thiocarbanilide when boiled with carbon disulphide, hydrocyanic acid escaping.

Thiocarbanilide is ordinarily prepared by boiling aniline and alcobolic potash with an excess of carbon disulphide.

\section{XXXV.-Analysis of Beet Root and Sorghum Cane. \\ By P. Cagamajor.}

In the United States, for the last twenty years, unany attempts have been made to manufacture sugar in a commercial way, from beet root and from the sorghum cane. Such attempts continue to be made at this day, and although there is no predicting what results 
may be obtained in the future, I believe that heretofore these attempts have not been successful.

There are many persons who believe that cane sugar can be made commercially from the sorghum cane, while others believe that beet root is preferable to sorghum. The partisans of beet and those of sorghum differ widely in their estimates of the comparative values of these two sources of sugar. There is only'one way of settling the question; which is to produce beet or sorghum which will yield good results. I have had occasion to examine several samples of beet root raised in the Northern States, and one of sorghum, and the results obtained were in all cases very unfavorable.

The object of this communication is to call attention to the processes by which we may ascertain beforeband the quantity of sugar which may be obtained from a given weight of bect root or of sorghum cane. It is nearly useless to try to ascertain the yield of a saccharine juice by experiments on a small scale, as such experiments never yield results analogous to those which can be obtained on a large scale with improved apparatus.

Beet root or sorghum cane can only be considered by the sugar manufacturer as so much raw material. To estimate the value of either, the manufacturer should follow exactly the course that a refiner follows to ascertain the commercial value of a raw sugar. A refiner knows that, from a given quantity $O_{\perp}$ ruw sugar, he can obtain a certain portion of pure or nearly pure sugar, and a certain quantity of molasses which will not yield any crystallizable sugar. 'Therofore, if he can ascertain the total quantity of sugar in raw sugar, and the portion which will remain in the molasses, the difference will be the pure sugar which he will obtain in refining.

It may be urged, that account should also be taken of a certain quantity of sugar lost during the process of refining, but this loss is due to causes entirely independert of the composition of any raw sugar under examination, and it should be left out of the calculation.

The composition of molasses from a well regulated sugar-house is pretty nearly constant. If raw cane sugars are used in a refinery, the composition of the molasses which will not yield any sugar crystals will be very nearly as follows for a syrup of density equal to $40^{\circ}$ Baumé:

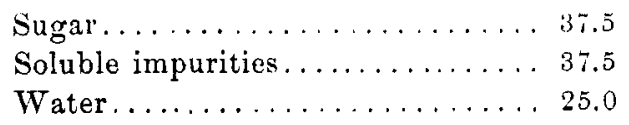


A refiner may obtain, as residue, a syrup containing a smaller percentage of sugar, but we may assume, for the purpose of comparing one sample of raw sugar with another, that the above will be the composition of the molasses obtained in refining.

For the sake of convenience in comparing one product with another in a sugar refinery, the composition of each product is sometimes stated in the dry state, or on the supposition that the product contains no water. Water is easily added, and it may be taken away by evaporation without affecting the quantity of pure sugar obtainable from a given commercial sugar. The quantity of sugar in a product reduced to dryness, compared to the total substances, is called coefficient of purity or quotient of purity.

Referring to the composition of the sugar-house molasses given above, we may see that its coefficient of purity is 50 , which means that in cane sugars 1 per cent. of soluble impurities prevents the crystallization of 1 per cent. of sugar.

It has been argued that the coefficient of purity of mulasses is not a safe guide for calculating the yield of pure sugar from a given sample of raw material.

There would be some foundation for this if the coefficient of purity of the molasses of a given refinery varied within very wide limits. The contrary is, however, the case. Thousands of tests, extending through several years, have convinced me that the coefficient of purity of molasses may be kept within very narrow limits. The coefficient is not always 50 . Some refiners prefer to keep it somewhat lower; but the coefficient 50 answers very well for calculating the quantity of pure sugar obtainable from a raw cane sugar.

To apply the above, let us suppose that we have a raw sugar whose coefficient of purity is 92 . The composition of the sugar, supposed dry, would be :

Sugar............................ 82

Impurities........................ 8

As 8 parts of impurities prevent the crystallization of 8 parts of sugar, the yield of the above dry sugar would be $92-8=84$ per cent.

The results which can be obtained from a given weight of beet root sugar are somewhat different from those which cane sugar will afford. A series of tests made in the principal sugar refineries of Paris show that the coefficient of purity of beet molasses seldom goes below 52, and is generally 55 . From this we may deduce the rule that in beet root sugar 1 per cent. of impurities prevent the crystallization of 1.2 per cent. of sugar. 
The composition of sorghum molasses, from which all the crystallizable sugar has been separated, has not been ascertained, as far as I know. From the analogy between sorghum and the sugar cane we may assume that, for sorghum sugar, 1 per cent. of soluble impurities prevents the erystallization of 1 per cent. of sugar.

The difficulty with beet root and sorghum cane grown in this country is principally that the coefficient of purity of their juices is not sufficiently high. Whenever a product is obtained whose coefticient of purity is high enough, the quantity of juice in a certain weight of roots or of cane may be ascertained, and this presents no difficulty. It, however, the coefficient of purity is not sufficiently high, there is no use in miaking further determinations, as there cannot be any profit in working a juice of low grade.

The coefficient of purity of a juice is determined very quickly and accurately by using a process which I first described in the American CHmist for October and November, 1873. This process is described more fully and in better form in the Moniteur Scientifique for March, 1877. In a late book on sugar analysis, published by Van Nostrand, it is said that this process is advisable when a balance is not at hand, but that it does not give as satisfactory results as Balling's process, which is mentioned as the dirent process.

As my process is in nse in most of the important refineries in the United States, I do not feel called upon to defend it. A constant use of it for several years shows that the results it gives agree within at least 1 per cent., and generally within less than $\frac{1}{2}$ per cent. This process is founded on this: That the pure sugar in 100 c.c. of a solution is given by multiplying the indication of the optical saccharometer by 0.26048 when a Ventzke instrument is used, or by 0.1635 for the Duboscq saccharometer. On the other hand, the total quantity of substance in solution is represented by the degree Balling, multiplied by the specific gravity.

The coefficient of purity is obtained by dividing the first product by the second. If we call the saccharimetric test $s$, the specific gravity $P$, and the Bailing degree $B$, we shall have-

$$
\text { Coefficient of purity }=\frac{S \times 0.26048}{B \times P^{-}}
$$

This may be written :

$$
\mathrm{S} \times \frac{0.26048}{\mathrm{~B} \times \mathrm{P}}
$$


If for every degree Balling we calculate the quantity

$\mathrm{B} \times \mathrm{P}$

we may form a table of factors by which to multiply the saccharimetric degree to obtain the coefficient of purity of a solution.

We may, instead of the Balling degree, determine the specific gravity, and for any specific gravity use the factor corresponding to

\subsection{8 \\ $\mathrm{B} \times \mathrm{P}$}

In the articles above referred to, tables are given for Balling degrees between $5^{\circ}$ and $15^{\circ}$, and for Geisler's specific gravity spindle, ranging from 1.095 to 1.1050 . For any other degree Balling, or any other specific gravity, the factors may be easily calculated from the formula given above.

The specific gravity may be determined by a balance, and the factor may be obtained by the following formula, in which the Balling degree does not enter:

$$
\frac{0.1}{P-1}
$$

in which $\mathrm{P}$ is the specitic gravity. This is for Ventzke's instrument For the Duboscq saccharometer, the factor is given by

$$
\frac{0.0628}{\mathrm{P}-1}
$$

'To test a lot of beets for coefficient of purity, the juice should be taken so as to represent the average juice of the lot. A wedgeshaped piece should be cut out of every root picked out for analysis. The thin edge of the wedge should be at the axis of the root, and the angle of each wedge should be the same for every piece. All the wedges may be rasped on a common grater, and the pulp obtained should be pressed in a cloth so as to obtain the juice. This is inmediately neutralized with lime, and heated, by which a thick black precipitate is obtained. The liquid, after filtration, is light and clear enough to be placed in the tube of the optical saccharometer. The density of the filtered liquid should be taken to determine the coeffcient of purity, as the juice of beets can always be defecated with a little lime.

The juice of sorghum cane is easily obtained by crushing with a hammer and wringing the cane with the hands, if no special apparatus is at hand. 
If the coefficient of purity of a juice is sufficiently high, we may in the next place determine the quantity of juice in a certain weight of the raw material.

The quantity of juice in the pulp obtained by rasping may be got at by drying a certain weight of the pulp and determining the water in the pulp by loss. We also determine the water in the juice by taking the difference between 100 and the Balling degree. As all the water in the pulp is in the juice, we have:

$$
\text { per cent. juice in the pulp }=\frac{\text { per cent. of water in the pulp. }}{\text { per cent. of water in the juice. }}
$$

As to what constitutes a sufficiently high coefficient of purity, this should be left to the judgment of the parties interested. I may, however, call to attention that in the North of France in 1879, a beet was not considered as fit to work for sugar, the coefficient of purity of whose juice was below 79. For 100 parts of dried juice the composition would be :

Pure sugar.......................... 79

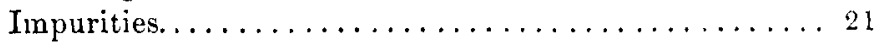

We have seen that in beet juice 1 part of impurities prevents the crystallization of 1.2 parts of sugar, therefore the yield would be :

$$
7^{\circ}-(21 \times 1.2)=79-25.2=53.8 \text { per cent. }
$$

I have had occasion to analyze several samples of beet roots grown in the United States. Those which gave the highest coefficient of purity were from Delaware, the coefficient of purity of the juice being 63 per cent. The yield of sngar of the dried juice would be

$$
63-(37 \times 1.2)=63-44.4=18.6 \text { per cent. }
$$

Most of the beet roots I tested had a coefficient of purity below 50 , and they could not, therefore, yield any sugar.

The coefficient of purity of the juice of a sorghum cane which I tested lately was 44 , and of course no sugar could be obtained from this juice.

It is almost unnecessary to state in conclusion, that the processes described are applicable to the sugar cane, and to other plants with saccharine juices. 